UDC 595.132.1]591.4:591.9(477)

\title{
MORPHOLOGICAL AND CHOROLOGICAL CHARACTERIZATION OF LONGIDORUS INTERMEDIUS KOZLOWSKA \& SEINHORST, 1979 FIRSTLY REPORTED FROM UKRAINE WITH THE COMMENTS ON LONGIDORUS ELONGATUS (DE MAN, 1876) THORNE \& SWANGER, 1936
}

\author{
S. A. Susulovska, J. V. Tsaryk \\ Ivan Franko National University of Lviv, 4, Hrushevskyi St., Lviv 79005, Ukraine \\ e-mail: solomija.s.a@gmail.com
}

Susulovska S. A., Tsaryk J. V. Morphological and chorological characterization of Longidorus intermedius Kozlowska \& Seinhorst, 1979 firstly reported from Ukraine with the comments on Longidorus elongatus (de Man, 1876) Thorne \& Swanger, 1936. Studia Biologica, 2018: 12(2); 53-62 • DOI: https://doi.org/10.30970/sbi.1202.552

The family Longidoridae Thorne, 1935 is a big taxon of Dorylaimina sub-family. Its species are ectoparasites of vascular plants inhabiting terrestrial biotopes. Longidoridae is one of the most homogeneous groups of Dorylaimina, which differs distinctly in morphology and biology from other dorylaimids, but its representatives structurally and ecologically are very similar to each other. Seventeen species of this family belonging to three genera were previously reported from the territory of Ukraine. This paper presents first record of Longidorus intermedius Kozlowska \& Seinhorst, 1979 and new records of closely related species Longidorus elongatus (de Man, 1876) Thorne \& Swanger, 1936 from Ukraine. Data on morphology, morphometrics and main differences between Ukrainian populations of these two species are provided. Ukrainian populations of $L$. intermedius are also compared in details with other previously reported populations of this species, and main differences are described. The presence of only three juvenile developmental stages in L. intermedius was confirmed. In model natural and anthropogenically altered localities on the territory of Opillia, the host preference of $L$. intermedius and $L$. elongatus was analyzed in detail. On this territory, $L$. intermedius is closely associated with Quercus robur L., so it can be used as an indicator for natural and semi-natural forests in which oak associations represent a primary vegetation type. On the contrary, L. elongatus populations were reported from the rhizosphere of a wide range of host plants. According to its chorological characters, L. intermedius can be also used in phylogeographical research in order to determine the formation ways of nemoral complexes of soil organisms after the Last Glacial Maximum.

Keywords: Longidorus intermedius, Longidorus elongatus, Ukraine, morphology, host-plant specialty

() 2018 S.A. Susulovska et al.; Published by the Ivan Franko National University of Lviv on behalf of Біологічні Студії / Studia Biologica. This is an Open Access article distributed under the terms of the Creative Commons Attribution License (http://www.budapestopenaccessinitiative.org/ and Creative Commons Attribution 4.0 License), which permits unrestricted reuse, distribution, and reproduction in any medium, provided the original work is properly cited.

ISSN 1996-4536 (print) • ISSN 2311-0783 (on-line) • Біологічні Студії / Studia Biologica • 2018 • Том 12/№2 • C. 53-62 


\section{INTRODUCTION}

The genus Longidorus Micoletzky, 1922 consists of more than 150 valid species which are ectoparasites of vascular plants inhabiting terrestrial biotopes [1]. Nine Longidorus species have been previously reported from Ukraine: Longidorus attenuatus Hooper, 1961, L. caespiticola Hooper, 1961, L. danuvii Barsi, Lamberti, De Luca, 2007, L. distinctus Lamberti, Choleva, Agostinelli, 1983, L. elongatus (de Man, 1876) Thorne \& Swanger, 1936, L. holovachovi Peneva, Susulovsky, Lazarova, 2009, L. poessneckensis Altherr, 1974, L. rubi Romanenko \& Tomilin in Romanenko, 1993 and L. sylphus Thorne, 1939. [11, 13, 14]. During recent survey, four populations of Longidorus genus were identified as Longidorus intermedius Kozlovska, Seinhorst, 1979. This is the first report of L. intermedius on the territory of Ukraine. This species was described from Netherlands and Germany from the rhizosphere of Quercus robur L. [7]. It was also previously reported from Belgium, Italy, Spain, countries of Balkan Peninsula and Central Europe and from the European part of Russia [2, 9, 10, 12]. Most populations of $L$. intermedius were collected in oak forests from the rhizosphere of Quercus spp. But it has also been reported on the rhyzosphere of some other plant species, for example olive Olea europea $\mathrm{L}$. in Spain and from the grasses in Belgium and Poland [4, 10]. Based on these data, Bulgarian nematologists concluded that this species is closely associated with oak forests and its potential distribution range may extend eastwards to the Dnipro River [10].

Longidorus elongatus is more widely distributed on the territory of Western Ukraine. It was previously detected in different types of oak and beech forests in Opillia, Subcarpathia and Carpathian mountains [6].

The objectives of this study were to determine main morphological and chorological characteristis of four populations of $L$. intermedius from the Western Ukraine and to describe morphological differences between $L$. intermedius and the most closely related and widely distributed species of $L$. elongatus.

\section{MATHERIALS AND METHODS}

Soil samples containing $L$. intermedius and $L$. elongatus were taken from natural and anthropogenically altered localities on the territory of Opillia and Zakarpattia in Ukraine. Localities and host-plants are presented in Table 1, 3. Nematodes were extracted from $500 \mathrm{~cm}^{3}$ of soil by modified sieving and decanting method [3]. Extracted specimens were heat killed, fixed in triethanolamine formalin (TAF), processed to glycerol by a slow evaporation method and mounted on permanent slides. Measurements and photos were made using Olympus BX 51 microscope with Nomarski differential interference contrast, equipped with a digital camera Olympus DP 72 and computer program Quick PHOTO MICRO 2.3.

\section{RESULTS AND DISCUSSION}

Longidorus intermedius Kozlowska \& Seinhorst, 1979 (Fig. 1, 2; Table 1, 2)

\section{Female}

Body of a medium length $-3.31-5.16 \mathrm{~mm}$, assuming C-shape or open spiral when heat relaxed. The anterior body end is conical, tapering gradually. Lip region slightly offset from the rest of the body, 5-6 $\mu \mathrm{m}$ high and 11-13 $\mu \mathrm{m}$ wide, rounded, slightly flattened frontally. Amphidial pouches large, stretching to about halfway of the distance from anterior end to guiding ring, symmetrically bilobed at the base. Odontostyle, odontophore and guide ring typical for genus. Oesophagus dorylaimoid with muscular bulb

ISSN 1996-4536 (print) • ISSN 2311-0783 (on-line) • Біологічні Студії / Studia Biologica • 2018 • Том 12/№2 • С. 53-62 
equaled $105(91-119) \times 21(17-25) \mu \mathrm{m}$. Oesophago-intestinal valve variable in shape, from conical to rounded. Vuvla a transverse slit situated almost equatorially, vagina 16 (13-19) $\mu \mathrm{m}$ wide and 26 (23-33) $\mu \mathrm{m}$ long occupying approximately half of the corresponding body width. Reproductive system amphidelphic, with equally developed genital branches 260 (205-314) and 240 (192-295) $\mu \mathrm{m}$ long, respectively. Prerectum of a variable length $344(233-483) \mu \mathrm{m}$, rectum $29(23-36) \mu \mathrm{m}$ long or as long as $3 / 4$ of anal body width. Tail dorsally convex, ventrally almost straight, bluntly conoid with a rounded terminus.

\section{Male}

Males are very rare and morphologically similar to female, with a posterior region of body more coiled. Body length $-3.31-4.22 \mu \mathrm{m}$. Spicules of a medium length -53.3 (51-56) $\mu \mathrm{m}$, very slightly curved ventrally. Lateral guiding pieces $16.9(16.8-17) \mu \mathrm{m}$ long, almost straight with slightly bifid distal ends. Adanal pair of supplements preceded by ventromedian row of 8-10 supplements. Tail ventrally more curved than in female, bluntly conoid with a rounded terminus.

Table 1. Data on localities, host plants and morphometrics of Ukrainian populations of $L$. intermedius. All measurements were done in $\mu \mathrm{m}$, except for body length $(\mathrm{mm})$ and in form: mean \pm standard deviation (range)

Таблиця 1. Дані про місцезнаходження, рослин-хазяїв і морфометрію українських популяцій L. intermedius. Усі виміри подано в мікрометрах, за винятком загальної довжини тіла (у міліметрах), у вигляді: середнє значення \pm стандартна похибка (межі мінливості)

\begin{tabular}{|c|c|c|c|c|c|c|}
\hline \multirow{2}{*}{$\begin{array}{l}\text { Location } \\
\text { and host plants } \\
\text { Character }\end{array}$} & \multirow[t]{2}{*}{$\begin{array}{c}\text { Lviv region, } \\
\text { Mykolaiv district, } \\
\text { Ustia village } \\
49^{\circ} 30^{\prime} 16.15^{\prime \prime} \mathrm{N} \\
23^{\circ} 54^{\prime} 54.23^{\prime \prime} \mathrm{E} \\
\text { Quercus robur L., } \\
\text { Acer campestre L. } \\
\text { female }\end{array}$} & \multicolumn{2}{|c|}{$\begin{array}{l}\text { Lviv region, Horodok } \\
\text { district, Liubin Malyj } \\
49^{\circ} 42^{\prime} 04.86^{\prime \prime} \mathrm{N} \\
23^{\circ} 45^{\prime} 57.90^{\prime \prime} \mathrm{E} \\
\text { Quercus robur L. }\end{array}$} & \multirow[t]{2}{*}{$\begin{array}{c}\text { Lviv, } \\
\text { Ivan Franko Park } \\
49^{\circ} 50^{\prime} 15.90^{\prime \prime} \mathrm{N} \\
24^{\circ} 01^{\prime} 06.26^{\prime \prime} \mathrm{E} \\
\text { Quercus robur L. } \\
\text { female }\end{array}$} & \multicolumn{2}{|c|}{$\begin{array}{c}\text { Zakarpattia } \\
\text { region, } \\
\text { Uzhhorod } \\
48^{\circ} 35^{\prime} 54.85^{\prime \prime} \mathrm{N} \\
22^{\circ} 22^{\prime} 11.43^{\prime \prime} \mathrm{E} \\
\text { Quercus robur L. }\end{array}$} \\
\hline & & male & female & & female & male \\
\hline$n$ & 31 & 2 & 13 & 11 & 14 & 1 \\
\hline$L$ & $\begin{array}{c}4.22 \pm 0.41 \\
(3.54-5.16)\end{array}$ & $\begin{array}{l}4.19 \\
3.76\end{array}$ & $\begin{array}{c}4.12 \pm 0.39 \\
(3.32-4.63)\end{array}$ & $\begin{array}{c}4.08 \pm 0.41 \\
(3.56-4.53)\end{array}$ & $\begin{array}{c}3.82 \pm 0.27 \\
(3.49-4.49)\end{array}$ & 3.31 \\
\hline a & $\begin{array}{c}79.3 \pm 6.24 \\
(66.1-97.4)\end{array}$ & $\begin{array}{l}83.8 \\
78.3\end{array}$ & $\begin{array}{c}80.8 \pm 4.46 \\
(75.4-92.5)\end{array}$ & $\begin{array}{c}77.5 \pm 5.81 \\
(67.1-87.0)\end{array}$ & $\begin{array}{c}75.5 \pm 1.84 \\
(72.5-78.5)\end{array}$ & 92.0 \\
\hline$b$ & $\begin{array}{l}11.2 \pm 1.76 \\
(9.2-17.0)\end{array}$ & $\begin{array}{l}9.6 \\
9.5\end{array}$ & $\begin{array}{c}10.2 \pm 1.12 \\
(8.4-12.7)\end{array}$ & $\begin{array}{c}10.7 \pm 1.84 \\
(8.4-13.3)\end{array}$ & $\begin{array}{l}10.3 \pm 1.07 \\
(7.9-12.4)\end{array}$ & 9.0 \\
\hline$c$ & $\begin{array}{c}111.1 \pm 15.42 \\
(78.7-139.6)\end{array}$ & $\begin{array}{c}107.5 \\
85.4\end{array}$ & $\begin{array}{c}98.3 \pm 9.31 \\
(80.9-115.7)\end{array}$ & $\begin{array}{l}107.1 \pm 14.58 \\
(83.0-128.3)\end{array}$ & $\begin{array}{c}100.8 \pm 8.71 \\
(89.7-115.2)\end{array}$ & 82.8 \\
\hline$c^{\prime}$ & $\begin{array}{c}1.09 \pm 0.13 \\
(0.91-1.44)\end{array}$ & $\begin{array}{l}1.1 \\
1.2\end{array}$ & $\begin{array}{c}1.22 \pm 0.05 \\
(1.12-1.29)\end{array}$ & $\begin{array}{c}1.15 \pm 0.12 \\
(0.92-1.31)\end{array}$ & $\begin{array}{l}1.12 \pm 0.09 \\
(1.0-1.3)\end{array}$ & 1.11 \\
\hline$d$ & $\begin{array}{l}2.7 \pm 0.20 \\
(2.4-3.9)\end{array}$ & $\begin{array}{l}2.8 \\
2.7\end{array}$ & $\begin{array}{l}2.8 \pm 0.16 \\
(2.5-3.1)\end{array}$ & $\begin{array}{l}2.9 \pm 0.15 \\
(2.7-3.2)\end{array}$ & $\begin{array}{l}2.6 \pm 0.18 \\
(2.4-3.0)\end{array}$ & 2.4 \\
\hline$d^{\prime}$ & $\begin{array}{l}1.9 \pm 0.12 \\
(1.6-2.1)\end{array}$ & $2 ; 2$ & $\begin{array}{l}2.0 \pm 0.12 \\
(1.8-2.3)\end{array}$ & $\begin{array}{l}2.1 \pm 0.13 \\
(1.8-2.3)\end{array}$ & $\begin{array}{l}2.0 \pm 0.12 \\
(1.8-2.3)\end{array}$ & 1.8 \\
\hline V / Spicules length & $\begin{array}{c}46.5 \pm 2.12 \\
(40.0-50.2)\end{array}$ & $\begin{array}{l}56 \\
53\end{array}$ & $\begin{array}{c}48.3 \pm 1.19 \\
(46.0-49.7)\end{array}$ & $\begin{array}{c}47.0 \pm 2.34 \\
(41.5-50.4)\end{array}$ & $\begin{array}{c}47.2 \pm 1.73 \\
(43.0-50.0)\end{array}$ & 51 \\
\hline Odontostylet length & $\begin{array}{c}111.2 \pm 3.1 \\
(106-122)\end{array}$ & $\begin{array}{l}110 \\
107\end{array}$ & $\begin{array}{l}108.8 \pm 2.9 \\
(105-114)\end{array}$ & $\begin{array}{r}111.2 \pm 1.9 \\
(109-114)\end{array}$ & $\begin{array}{l}107.9 \pm 2.6 \\
(106-112)\end{array}$ & 106 \\
\hline
\end{tabular}

ISSN 1996-4536 (print) • ISSN 2311-0783 (on-line) • Біологічні Студії / Studia Biologica • 2018 • Том 12/№2 • C. 53-62 


\begin{tabular}{lcccccc} 
& \multicolumn{3}{c}{ End of the Table 1 } \\
\hline Odontophore length & $63.8 \pm 3.5$ & $63 ;$ & $65.2 \pm 2.4$ & $63.3 \pm 3.0$ & $60.9 \pm 2.2$ & 53 \\
& $(57-72)$ & 67 & $(61-69)$ & $(58-68)$ & $(58-65)$ & \\
Total stylet length & $175.0 \pm 5.2$ & $173 ;$ & $174.0 \pm 3.4$ & $174.5 \pm 3.2$ & $168.8 \pm 3.8$ & 159 \\
& $(167-188)$ & 174 & $(168-181)$ & $(171-182)$ & $(164-177)$ & \\
Anterior and to guide ring & $34.0 \pm 1.1$ & $34 ;$ & $32.4 \pm 1.0$ & $33.7 \pm 1.3$ & $31.0 \pm 1.0$ & 29 \\
& $(32-36)$ & 32 & $(31-34)$ & $(31-35)$ & $(29-33)$ & \\
Pharyngeal bulb length & $106.4 \pm 6.0$ & $113 ;$ & $107.2 \pm 5.7$ & $106.3 \pm 6.5$ & $99.9 \pm 5.5$ & 98 \\
& $(93-119)$ & 105 & $(99-118)$ & $(93-114)$ & $(91-110)$ & \\
Pharyngeal bulb width & $20.7 \pm 1.4$ & $21 ;$ & $20.5 \pm 1.7$ & $21.6 \pm 1.6$ & $21.6 \pm 1.6$ & 21 \\
Tail length & $(18-23)$ & 22 & $(17-23)$ & $(18-24)$ & $(19-25)$ & \\
& $38.4 \pm 3.9$ & $39 ;$ & $42.0 \pm 2.5$ & $38.5 \pm 4.0$ & $38.1 \pm 3.2$ & 40 \\
Hyaline part of tail length & $(31-46)$ & 44 & $(38-47)$ & $(32-46)$ & $(34-43)$ & \\
Width at level of: & $12.1 \pm 1.1$ & $14 ;$ & $11.8 \pm 0.9$ & $12.0 \pm 1.2$ & $10.5 \pm 1.5$ & 10 \\
lips & $(10-15)$ & 13 & $(11-14)$ & $(10-14)$ & $(7-13)$ & \\
& & & & & & \\
guide ring & $12.6 \pm 0.9$ & $12 ;$ & $11.8 \pm 0.7$ & $11.6 \pm 0.5$ & $11.8 \pm 0.8$ & 12 \\
& $(11-14)$ & 12 & $(11-13)$ & $(11-12)$ & $(11-13)$ & \\
base of pharynx & $24.6 \pm 1.0$ & $24 ;$ & $23.7 \pm 0.8$ & $24.2 \pm 2.3$ & $23.6 \pm 1.1$ & 22 \\
& $(23-27)$ & 24 & $(22-25)$ & $(22-26)$ & $(22-25)$ & 22 \\
vulva or mid-body & $44.7 \pm 2.2$ & $44 ;$ & $43.5 \pm 2.6$ & $44.2 \pm 2.1$ & $42.9 \pm 2.7$ & 42 \\
& $(41-49)$ & 43 & $(39-48)$ & $(42-49)$ & $(38-48)$ & \\
anus & $53.3 \pm 5.1$ & $50 ;$ & $51.0 \pm 3.9$ & $52.7 \pm 3.9$ & $50.6 \pm 3.8$ & 46 \\
& $(46-65)$ & 48 & $(44-57)$ & $(48-60)$ & $(47-60)$ & \\
& $35.1 \pm 2.6$ & $35 ;$ & $34.5 \pm 2.1$ & $33.5 \pm 2.7$ & $34.0 \pm 2.2$ & 36 \\
\hline
\end{tabular}

\section{Juveniles (Table 2)}

The analysis of morphometrics revealed presence of only three juvenile developmental stages, same as in previous publications [2, 7, 9, 10]. Morphologically juveniles are similar to adults. Juvenile stages can be clearly distinguished by the body length and the length of functional and replacement odontostyle. Tail length of juveniles doesn't change significantly during their development, but $\mathrm{J} 1$ tail is characterized by a digitate terminus.

All four Ukrainian populations of $L$. intermedius are very similar to each other and to previously described populations, but some differences were revealed during the analysis. In comparison to type population from the Netherlands, they have higher values of $b$ (10.1 (8.4-12.7); 11.2 (9.2-17.0); 10.7 (8.4-13.3); 10.3 (7.9-12.4) vs 9-10) and $c$ (98.3 (80.9-115.7); 111.1 (78.6-139.6); 107.1 (83.0-128.3); 100.8 (89.7-115.2) vs $87-109)$ indices, more posterior position of guiding ring (29-36 vs 27-34 $\mu \mathrm{m})$ and wider lip region (11-14 vs 11-12 $\mu \mathrm{m})$ [7].

Studies populations are most similar to populations from Bulgaria, but differ by slightly shorter tail (mean $38.1 ; 38.4 ; 38.5 ; 42$ vs $41 ; 42 ; 43 ; 43 ; 44 ; 47 \mu \mathrm{m}$ ) and lower $c^{\prime}$ value (mean $1.09 ; 1.12 ; 1.15 ; 1.22$ vs $1.2 ; 1.2 ; 1.2 ; 1.3 ; 1.3 ; 1.4$ ) [10]. They are more similar to Italian, Spanish and Czech populations [9, 10].

Previously, few male specimens were reported from the countries of Balkan Peninsula: Bulgaria, Croatia, Bosnia and Herzegovina and Serbia [2, 10]. Males from Ukrainian populations differ from the Bulgarian ones by more slender body (index a values: 78-92 vs 66-74), slightly longer spicules (51-56 vs 41-51 $\mu \mathrm{m})$, and different number of supplements (8-10 vs 6-7) [10]. In comparison to males from Croatia, Bosnia and Herzegovina and Serbia, studied male specimens have slightly shorter body (3.35-4.19 vs $3.31-4.72 \mu \mathrm{m})$ and tail (39-44 vs $42-52 \mu \mathrm{m})$, but slightly longer spicules (51-56 vs $43-52 \mu \mathrm{m})$ and higher number of supplements (8-10 vs 4-8) [2].

ISSN 1996-4536 (print) • ISSN 2311-0783 (on-line) • Біологічні Студії / Studia Biologica • 2018 • Том 12/№2 • С. 53-62 

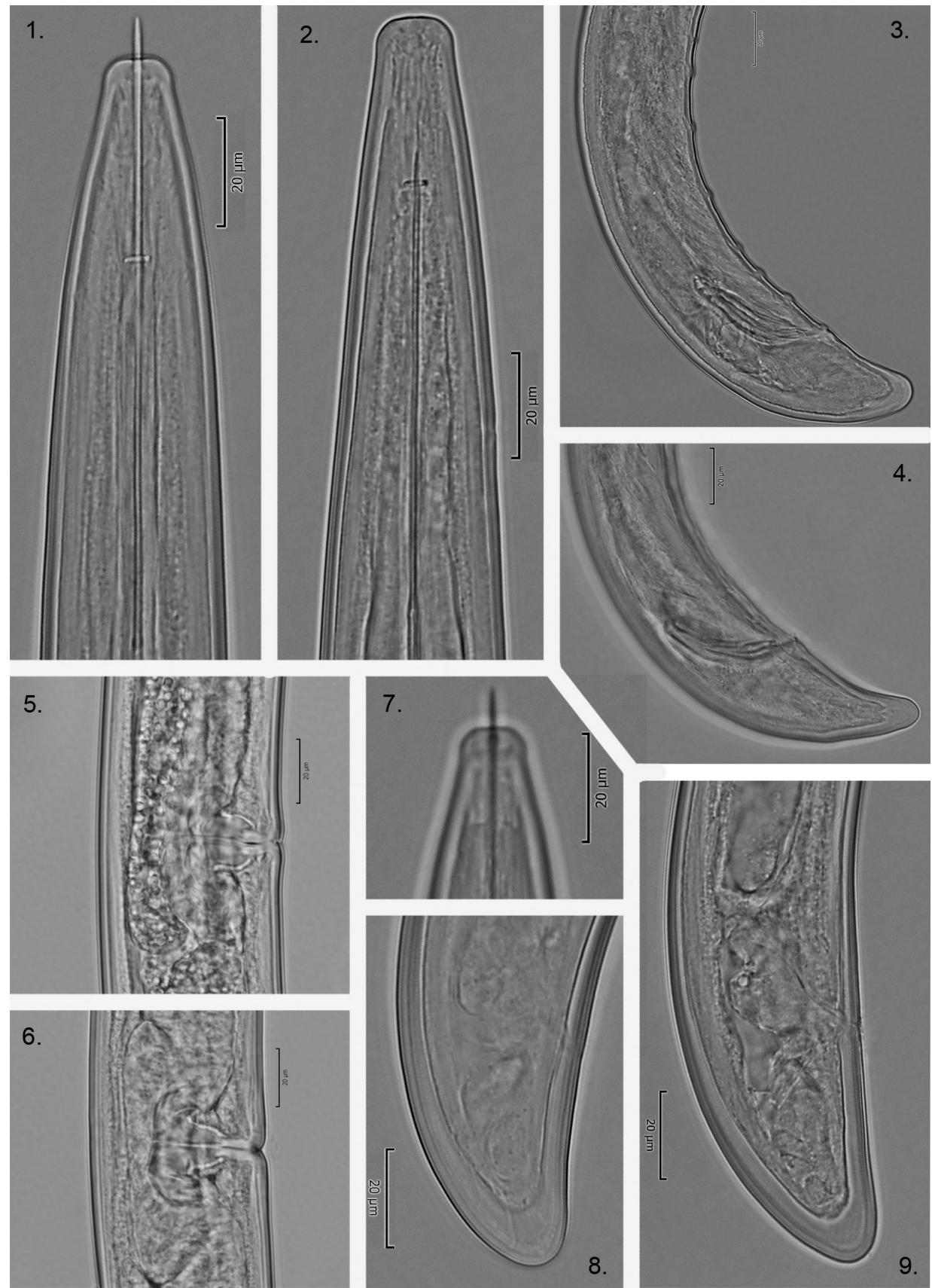

4.

Fig. 1. Morphology of Longidorus intermedius $(1,3,5,7,8)$ and $L$. elongatus $(2,4,6,9)$ from Ukraine: 1,2 female anterior regions; 3, 4-male tail regions; 5, 6-vulval regions; 7 -female anterior region with focus on aphidial fovea; 8, 9 - female tails

Рис. 1. Морфологічні особливості українських популяцій Longidorus intermedius $(1,3,5,7,8)$ і L. elongatus $(2,4,6,9): 1,2$ - передня частина тіла самок; 3, 4 - хвостова частина тіла самців; 5, 6 - вульва; 7 - передня частина тіла самки з акцентом на амфід; 8, 9 - хвости самок

ISSN 1996-4536 (print) • ISSN 2311-0783 (on-line) • Біологічні Студії / Studia Biologica • 2018 • Том 12/№2 • С. 53-62 

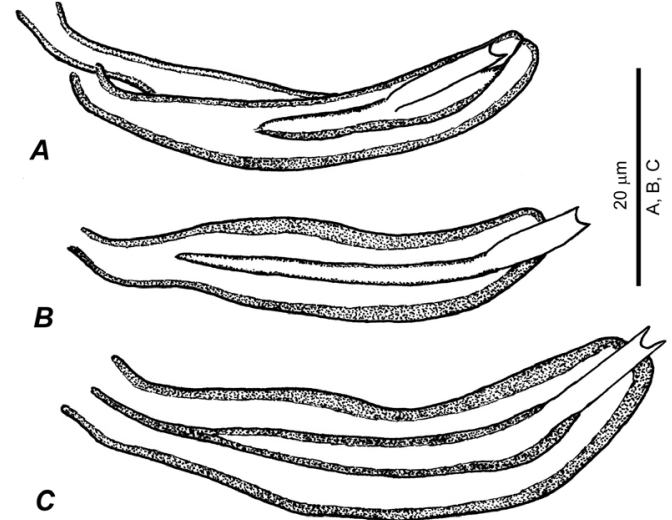

Fig. 2. Morphology of male spicules of Longidorus intermedius $(A, B)$ and $L$. elongatus $(C)$ from Ukraine (Lviv region)

Рис. 2. Морфологія спікул самців Longidorus intermedius $(A, B)$ i $L$. elongatus $(C)$, зібраних на території України (Львівська область)

Table 2. Data on morphometrics of Longidorus intermedius juveniles from the floodplain forest near Ustia (Lviv region, Ukraine). All measurements are in $\mu \mathrm{m}$, except for body length (in $\mathrm{mm}$ ) and in form: mean \pm standard deviation (range)

Таблиця 2. Морфометричні параметри личинок популяції Longidorus intermedius із заплавного лісу поблизу с. Устя (Львівська область, Україна). Усі виміри подано в мікрометрах, за винятком загальної довжини тіла (у міліметрах), у вигляді: середнє значення \pm стандартна похибка (межі мінливості)

\begin{tabular}{|c|c|c|c|}
\hline Character & J2 & J3 & J4 \\
\hline $\mathrm{n}$ & 17 & 25 & 17 \\
\hline$L$ & $1.24 \pm 0.53(1.16-1.34)$ & $1.92 \pm 0.20(1.62-2.30)$ & $2.89 \pm 0.20(2.44-3.17)$ \\
\hline$a$ & $54.3 \pm 2.03(50.3-57.8)$ & $61.8 \pm 4.59(47.7-69.6)$ & $71.4 \pm 3.99(66.0-78.1)$ \\
\hline$b$ & $4.8 \pm 0.91(4.3-8.1)$ & $6.4 \pm 0.52(5.5-7.2)$ & $8.2 \pm 0.79(6.9-9.9)$ \\
\hline$c$ & $26.9 \pm 1.25(24.6-29.6)$ & $41.6 \pm 3.96(35.1-48.9)$ & $64.0 \pm 5.73(55.5-78.4)$ \\
\hline$c^{\prime}$ & $3.11 \pm 0.18(2.81-3.43)$ & $2.12 \pm 0.17(1.90-2.47)$ & $1.50 \pm 0.10(1.23-1.66)$ \\
\hline$d$ & $2.9 \pm 0.22(2.5-3.3)$ & $2.8 \pm 0.22(2.5-3.3)$ & $2.8 \pm 0.19(2.5-3.3)$ \\
\hline$d^{\prime}$ & $2.1 \pm 0.19(1.8-2.5)$ & $2.0 \pm 0.15(1.7-2.3)$ & $2.0 \pm 0.11(1.9-2.3)$ \\
\hline Odontostylet length & $74.0 \pm 2.0(70-78)$ & $81.6 \pm 1.6(78-85)$ & $93.8 \pm 2.8(87-98)$ \\
\hline Odontophore length & $43.7 \pm 2.8(40-51)$ & $50.5 \pm 2.5(44-54)$ & $59.9 \pm 3.0(55-65)$ \\
\hline Total stylet length & $117.8 \pm 3.9(112-126)$ & $132.1 \pm 3.0(124-138)$ & $153.7 \pm 4.1(147-162)$ \\
\hline Replacement odontostylet length & $81.2 \pm 2.7(78-88)$ & $96.9 \pm 2.9(90-102)$ & $112.6 \pm 3.4(107-118)$ \\
\hline Anterior end to guide ring & $19.5 \pm 0.9(18-21)$ & $24.8 \pm 1.2(23-28)$ & $28.4 \pm 1.2(26-30)$ \\
\hline Pharyngeal bulb length & $62.5 \pm 3.4(55-69)$ & $74.4 \pm 4.3(68-86)$ & $85.8 \pm 5.2(78-95)$ \\
\hline Pharyngeal bulb width & $12.6 \pm 1.2(10-14)$ & $16.0 \pm 1.2(13-18)$ & $18.3 \pm 1.0(16-20)$ \\
\hline Tail length & $46.4 \pm 1.7(43-49)$ & $46.2 \pm 3.5(40-53)$ & $45.3 \pm 3.8(37-51)$ \\
\hline Hyaline part of tail length & $9.5 \pm 1.5(6-13)$ & $9.0 \pm 1.0(8-11)$ & $9.5 \pm 0.9(8-11)$ \\
\hline \multicolumn{4}{|l|}{ Width at level of: } \\
\hline lips & $6.9 \pm 0.7(6-8)$ & $8.8 \pm 0.7(8-10)$ & $10.0 \pm 0.4(9-11)$ \\
\hline guide ring & $14.1 \pm 0.8(13-16)$ & $17.1 \pm 1.0(15-19)$ & $20.4 \pm 0.7(19-21)$ \\
\hline base of pharynx & $21.8 \pm 0.8(20-23)$ & $29.4 \pm 2.2(26-34)$ & $36.7 \pm 1.8(33-40)$ \\
\hline mid-body & $22.9 \pm 0.9(22-25)$ & $31.0 \pm 2.4(26-35)$ & $40.5 \pm 2.7(36-46)$ \\
\hline anus & $14.9 \pm 0.7(14-16)$ & $19.9 \pm 2.7(15-25)$ & $30.2 \pm 1.7(27-33)$ \\
\hline
\end{tabular}

ISSN 1996-4536 (print) •ISSN 2311-0783 (on-line) • Біологічні Студії / Studia Biologica • 2018 • Том 12/№2 • C. 53-62 
Juveniles from Ustia (Lviv region, Ukraine) population are very similar to those from Dedevo-Rhodopi Mountain population, Bulgaria [10], but have some difference from juveniles of Czech population. They differ by slightly longer tail (JI - 46.4 (43-49) vs 43 (36-52) $\mu \mathrm{m}$; JII - 46.2 (40-53) vs 43 (39-51) $\mu \mathrm{m}$; JIII - 45.3 (37-51) vs 42 (37-49) $\mu \mathrm{m})$ and higher $c^{\prime}$ values ( $\mathrm{JI}-3.11(2.81-3.49)$ vs $2.83(1.96-3.36)$; JII $-2.12(1.90-$ $2.47)$ vs 1.98 (1.78-2.25); JIII - 1.5 (1.23-1.66) vs $1.47(1.16-1.88)$ ) in all juvenile developmental stages, longer replacement odontostyle in JII (96.9 (90-102) vs 91 (8499) $\mu \mathrm{m})$ and higher a values (71.4 (66.0-78.1) vs 64.9 (57.8-77.1)) in JIII [9].

Table 3. Data on localities, host plants and morphometrics of two Ukrainian populations of $L$. elongatus. All measurements were conducted in $\mu \mathrm{m}$, except for body length $(\mathrm{mm})$ and in form: mean \pm standard deviation (range)

Таблиця 3. Дані про місцезнаходження, рослин-хазяїв і морфометрію двох українських популяцій L. elongatus. Усі виміри подано в мікрометрах, за винятком загальної довжини тіла (у міліметрах), у вигляді: середнє значення \pm стандартна похибка (межі мінливості)

\begin{tabular}{|c|c|c|c|}
\hline Location and host plant & \multicolumn{2}{|c|}{$\begin{array}{l}\text { Lviv, garden square "Enzyme" } \\
49^{\circ} 50^{\prime} 02.20^{\prime \prime} \mathrm{N} 24^{\circ} 05^{\prime} 14.13^{\prime \prime} \mathrm{E} \\
\text { Acer sp. }\end{array}$} & \multirow{2}{*}{$\begin{array}{l}\text { Lviv region, Mykolaiv district, } \\
\text { Ustia village } \\
49^{\circ} 30^{\prime} 18.85^{\prime \prime} \mathrm{N} 23^{\circ} 54^{\prime} 56.95^{\prime \prime} \mathrm{E} \\
\text { Salix sp. } \\
\text { female }\end{array}$} \\
\hline Character & female & male & \\
\hline $\mathrm{n}$ & 30 & 4 & 20 \\
\hline$L$ & $5.25 \pm 0.56(4.52-6.90)$ & $5.15(4.39-5.74)$ & $5.14 \pm 0.40(4.41-6.04)$ \\
\hline$a$ & $97.5 \pm 5.66(86.9-109.4)$ & $101.2(95.3-108.3)$ & $95.6 \pm 6.04(83.2-108.6)$ \\
\hline$b$ & $12.6 \pm 1.74(10.4-18.6)$ & $11.8(9.3-13.4)$ & $12.1 \pm 1.12(10.4-14.7)$ \\
\hline$c$ & $112.5 \pm 16.79(86.6-160.3)$ & $94.3(78.3-110.8)$ & $120.1 \pm 12.01(101.4-137.7)$ \\
\hline$c^{\prime}$ & $1.19 \pm 0.10(1.00-1.39)$ & $1.40(1.23-1.56)$ & $1.15 \pm 0.07(0.97-1.28)$ \\
\hline$d$ & $2.2 \pm 0.13(1.9-2.5)$ & $2.2(2.1-2.4)$ & $2.3 \pm 0.12(2.1-2.5)$ \\
\hline$d^{\prime}$ & $1.6 \pm 0.08(1.4-1.7)$ & $1.5(1.4-1.6)$ & $1.6 \pm 0.07(1.5-1.7)$ \\
\hline V / Spicules length & $48.5 \pm 1.92(44.4-52.1)$ & $59(47-63)$ & $48.6 \pm 2.09(44.5-52.7)$ \\
\hline Odontostylet length & $85.5 \pm 6.3(77-99)$ & $82.5(80-89)$ & $91.2 \pm 4.1(85-100)$ \\
\hline Odontophore length & $65.6 \pm 3.1(59-73)$ & $68(67-69)$ & $60.2 \pm 4.0(51-65)$ \\
\hline Total stylet length & $148.9 \pm 43.5(138-165)$ & $150.5(147-158)$ & $151.1 \pm 5.7(149-159)$ \\
\hline Anterior end to guide ring & $32.0 \pm 1.1(30-34)$ & $32.8(31-34)$ & $32.2 \pm 1.1(30-35)$ \\
\hline Pharyngeal bulb length & $113.4 \pm 5.9(104-125)$ & $115(107-120)$ & $117.2 \pm 8.3(104-136)$ \\
\hline Pharyngeal bulb width & $20.2 \pm 1.2(18-22)$ & $18.7(17-21)$ & $21.8 \pm 1.8(19-24)$ \\
\hline Tail length & $47.0 \pm 3.8(40-55)$ & $55.3(48-67)$ & $43.0 \pm 3.6(35-50)$ \\
\hline Hyaline part of tail length & $11.3 \pm 1.7(9-16)$ & $13.5(12-16)$ & $12.3 \pm 1.0(11-14)$ \\
\hline \multicolumn{4}{|l|}{ Width at level of: } \\
\hline lips & $14.5 \pm 0.8(13-16)$ & $14.8(14-15)$ & $14.1 \pm 0.6(13-15)$ \\
\hline guide ring & $22.7 \pm 0.7(22-24)$ & $22(21-23)$ & $22.6 \pm 0.8(20-24)$ \\
\hline base of pharynx & $44.3 \pm 1.7(40-47)$ & $43.5(41-46)$ & $45.3 \pm 2.1(41-49)$ \\
\hline vulva or mid-body & $53.7 \pm 3.6(48-63)$ & $50.8(45-54)$ & $53.8 \pm 3.2(47-59)$ \\
\hline anus & $39.6 \pm 1.5(37-43)$ & $39.5(35-43)$ & $37.3 \pm 2.0(34-41)$ \\
\hline
\end{tabular}

ISSN 1996-4536 (print) • ISSN 2311-0783 (on-line) • Біологічні Студії / Studia Biologica • 2018 • Том 12/№2 • С. 53-62 
Longidorus elongatus (de Man, 1876) Thorne \& Swanger, 1936 (Fig. 1, 2; Table 3)

During this study new populations of Longidorus elongatus were identified. This species is more widely distributed in the Western Ukraine. It was previously reported from the basin of Dnister river, but no morphological data for those populations are available [6]. In this paper, for the first time we provide morphometrics of two Ukrainian populations of $L$. elongatus. This species morphologically is most similar to $L$. intermedius and closely related molecularly $[4,7,12]$. As these two species were found in adjacent localities, it is important to distinguish them from each other in order to avoid misidentification.

Main differences between Ukrainian populations of $L$. intermedius and $L$. elongatus are:

- Body length (3.32-5.16 vs $4.41-6.90 \mathrm{~mm})$

- Odontostyle length (105-122 vs 77-100 $\mu \mathrm{m})$

- Form of lip region (more rounded in L. intermedius)

- Length of genital branches (192-314 vs 311-626 $\mu \mathrm{m}$ )

- Form and length of spicules in males (slightly curved ventrally, 51-56 $\mu \mathrm{m}$ vs arcuate, 47-63 $\mu \mathrm{m}$; in L. elongatus lateral guiding pieces are shorter (14-15 $\mu \mathrm{m})$ and more distinctly bifid) (Fig. 2)

L. intermedius and L. elongatus also differ in their host plant specialty, which was analyzed in two model localities: floodplain forest near Ustia village and the city of Lviv. In the floodplain forest, most $L$. intermedius specimens were found in the rizosphere of Quercus robur L. and only in one case - from Acer campestre L. Samples were also taken from the rizosphere of Tilia cordata Mill., Fraxinus excelsior L., Euonymus europaeus L., Acer negundo L. and Corylus avellana L., but no L. intermedius specimens were found. L. elongatus specimens, unlike $L$. intermedius, were present only in samples from the rizosphere of Salix alba L., S. fragilis L., Populus sp. and grasses in the riparian zone of Dnister river, however never from oak tree in the middle part of the forest.

On the territory of Lviv only one population of $L$. intermedius was identified from the rizosphere of tricentennial oak tree (Quercus robur L.) in Ivan Franko Park - one of the oldest parks which was set up in the end of $16^{\text {th }}$ century in Lviv [8]. This finding may reveal that old oak trees currently growing on the territory of this park in spite of its numerous reconstructions are the elements of natural forests which had been growing in this region in the past. On the contrary, L. elongatus populations were found in many parks in different parts of the city in the rhyzosphere of Acer platanoides L., A. pseudoplatanus L., Fagus sylvatica L., Salix spp., some fruit trees (Malus domestica Borkh, Pyrus sp., Prunus cerasifera Ehrh, Prunus avium L.) and grasses.

The results of study of $L$. intermedius on the territory of the Western Ukraine show that this species is closely associated with natural oak forests. Four populations of $L$. intermedius were found on this territory, and three of them were collected in old oak forests and the fourth - from the rizosphere of tricentennial oak tree in the city park in Lviv. These data confirm a hypothesis of Bulgarian nematologists that this species, together with other nematode species, belongs to nemoral horological complex. According to this characteristic, $L$. intermedius can be used as an indicator for natural and semi-natural forests in which oak associations represent a primary vegetation type [10]. Taking into account the chorological characters, this species can be also used in the phylogeographical study in order to determine the formation ways of nemoral complexes of soil organisms after the Last Glacial Maximum (24.0-17.0 kyr BP). That is why distribution study of this species on the whole territory of Ukraine is of a great significance.

ISSN 1996-4536 (print) • ISSN 2311-0783 (on-line) • Біологічні Студії / Studia Biologica • 2018 • Том 12/№2 • С. 53-62 


\section{CONCLUSION}

The results of morphological and morphometrical analysis show that the Ukrainian populations of $L$. intermedius are similar to type and other previously reported populations, however differ in some characters and morphometric parameters. Differences between $L$. intermedius and closely related species $L$. elongatus are provided. In addition, currant research reveals that $L$. intermedius is closely associated with Quercus robur $L$. and can be used for indication of natural and semi-natural forests in which oak associations represent a primary vegetation type and in phylogeographical study of the formation ways of nemoral complexes of soil organisms.

1. Archidona-Yuste A., Navas-Cortés J.A., Cantalapiedra-Navarrete C., Palomares-Rius J.E. \& Castillo $P$. Unravelling the biodiversity and molecular phylogeny of needle nematodes of the genus Longidorus (Nematoda: Longidoridae) in olive and a description of six new species. PLoS ONE, 2016; 11(1): e0147689. [DOI: 10.1371/journal.pone.0147689].

2. Barsi L., Lamberti F. Morphometric variation and juvenile stages of Longidorus intermedius Kozlowska \& Seinhorst, 1979 (Nematoda: Dorylaimida) from the territory of the former Yugoslavia. Russian Journal of Nematology, 2004; 12(2): 107-114.

3. Brown D.J.F., Boag B. An examination of methods used to extract virus-vector nematodes (Nematoda: Longidoridae and Trichodoridae) from soil samples. Nematologia Mediterranea, 1988; 16: 93-99.

4. Gutierrez-Gutierrez C., Cantanalapiedra-Navarette C., Montes-Borrego M., Palomares-Rius J.E., Castillo P. Molecular phylogeny of the nematode genus Longidorus (Nematoda: Longidoridae) with description of three new species. Zoological Journal of the Linnean Society, 2013; 167: 473-500. [DOI: 10.1111/zoj.12019].

5. Hooper D.J. A redescription of Longidorus elongatus (De Man, 1876) Thorne \& Swanger, 1936 (Nematoda, Dorylaimidae) and description of five new species of Longidorus from Great Britain. Nematologica, 1961; 6: 237-257. [DOI: 10.1163/187529261X00072].

6. Kozlovsky M.P. Phytonematodes of terrestrial ecosystems of the Carpathian region. Lviv, 2009. 316 p. (In Ukrainian).

7. Kozlowska J., Seinhorst J. W. Longidorus elongatus and closely related species in the Netherlands and Lower Saxony (Germany), with the description of two new species, L. cylindricaudatus and L. intermedius (Nematoda: Dorylaimida). Nematologica, 1979; 25: 42-53. [DOI: 10.1163/187529279X00361].

8. Kucheriavyi V. P. Gardens and parks of Lviv. Lviv: Svit, 2008. 360 p. (In Ukrainian).

9. Kumari S., Chaloupkova M., Jokes M. First record of Longidorus intermedius Kozlowska and Seinhorst, 1979 (Nematoda: Longidorudae) from the Czech Republic. Helminthologia, 2006; 43(2): 122-124. [DOI: https://doi.org/10.2478/s11687-006-0023-z].

10. Peneva V., Loof P.A.A., Penev L.D. \& Brown D.J.F. Description of the male and first-stage juvenile of Longidorus intermedius Kozlowska \& Seinhorst, 1979 (Nematoda: Dorylaimida), and notes on its morphology and distribution. Systematic Parasitology, 2001; 49: 127-137. [DOI: https://doi.org/10.1023/A:1010608418412].

11. Peneva V.K., Susulovsky A., Lazarova S. Description of Longidorus holovachovi sp. n. (Nematoda: Dorulaimida) and Xiphinema sp., a member of Xiphinema americanum group from Ukraine. Russian Journal of Nematology, 2009; 17(2): 115-126.

12. Subbotin S.A., Rogozhin E.A., Chizhov V.N. Molecular characterization and diagnostics of some Longidorus species (Nematoda: Longidoridae) from Russia and other countries using rRNA genes. European Journal of Plant Pathology, 2014; 138(2): 377-390. [DOI: https:// doi.org/10.1007/s10658-013-0338-9].

13. Susulovska S., Castillo P., Archidona-Yuste A. First Reports, Morphological, and Molecular Characterization of Longidorus caespiticola and Longidorus poessneckensis (Nematoda: Longidoridae) from Ukraine. Journal of Nematology, 2017, 49(4), 396-402. 
14. Susulovska S., Susulovsky A., Kornobis F.W. Morphometrical and molecular data on plant parasitic nematodes Longidorus attenuatus Hooper, 1961 and L. danuvii Barsi et al., 2007 (Nematoda: Longidoridae) reported from Ukraine for the first time. Helminthologia, 2016; 53(4): 396-400. [DOI: 10.1515/helmin-2016-0040].

\title{
МОРФОЛОГІЯ ТА ХОРОЛОГІЯ НОВОГО ДЛЯ ФАУНИ УКРАЇНИ ВИДУ LONGIDORUS INTERMEDIUS KOZLOWSKA \& SEINHORST, 1979 I МОРФОЛОГІЧНІ ДАНІ УКРАЇНСЬКИХ ПОПУЛЯЦІЙ LONGIDORUS ELONGATUS (DE MAN, 1876) THORNE \& SWANGER, 1936
}

\author{
С. А. Сусуловська, Й. В. Царик \\ Львівський національний університет імені Івана Франка \\ вул. Грушевського, 4, Львів 79005, Україна \\ e-mail: solomija.s.a@gmail.com
}

Родина Longidoridae Thorne, 1935 - це один із найчисленніших таксонів підряду Dorylaimina (Enoplia), представники якого населяють наземні біотопи і $€$ ектопаразитами вищих рослин. Лонгідориди складають одну з найбільш гомогенних груп Dorylaimina, яка, з одного боку, морфологічно і біологічно чітко відокремлена від усіх інших груп дорилаймін, а з іншого - ії̈ представники структурно і екологічно дуже близькі між собою. Раніше на території України було виявлено 17 видів цієї родини, що належать до родів Longidorus, Paralongidorus і Xiphinema. У статті детально проаналізовано морфологічні й морфометричні особливості Longidorus intermedius Kozlowska \& Seinhorst, 1979, що вперше зібраний на території України. Проведено порівняння українських популяцій цього виду із типовою та іншими раніше відомими популяціями, а всі відмінності детально описано. Підтверджено наявність у L. intermedius лише трьох личинкових стадій. Встановлено основні відмінності між українськими популяціями L. intermedius і близькоспорідненого виду Longidorus elongatus (de Man, 1876) Thorne \& Swanger, 1936. Уперше наводиться морфометрія для популяцій L. elongatus, зібраних в Україні. На модельних, природній і антропогенно зміненій ділянках на території Опілля проаналізовано гостальну специфічність обох видів. Встановлено, що L. intermedius тісно асоційований із дубом звичайним (Quercus robur L.) і може слугувати індикатором для природних та напівприродних лісів, у яких угруповання дуба $є$ первинним типом рослинності. На відміну від попереднього виду, L. elongatus було виявлено у пробах ґрунту із ризосфери багатьох видів рослин, проте жодного разу з дуба. Запропоновано використання L. intermedius як модельного об'єкта у філогеографічних дослідженнях, щоби встановити способи фрормування неморальних комплексів ґрунтових організмів після останнього льодовикового максимуму.

Ключові слова: Longidorus intermedius, Longidorus elongatus, Україна, морфологія, гостальна специфічність

Одержано: 20.03.2018

ISSN 1996-4536 (print) • ISSN 2311-0783 (on-line) • Біологічні Студії / Studia Biologica • 2018 • Том 12/№2 • C. 53-62 